\title{
Atomistic simulation of $\mathrm{CeO}_{2}$ surface hydroxylation: implications for glass polishing
}

\author{
Christopher R. Stanek • Averyl H. H. Tan • \\ Scott L. Owens $\cdot$ Robin W. Grimes
}

Received: 6 February 2008/Accepted: 20 March 2008/Published online: 25 April 2008

(C) Springer Science+Business Media, LLC 2008

\begin{abstract}
Atomistic simulation techniques have been used to investigate the dissociative adsorption of water on the (110), (111), and (100) low index surfaces of $\mathrm{CeO}_{2}$, as well as a so-called "trench" surface configuration. Several different coverages of water have been considered to better understand how the hydroxylation process progresses. Hydroxylation energies and surface energies of $\mathrm{CeO}_{2}$ calculated via atomistic simulations are compared to similar results for other fluorite oxides. Finally, the modification of $\mathrm{CeO}_{2}$ crystallite morphology in the presence of water is predicted from the changes in surface energy and the implications of these morphological changes for glass polishing are discussed.
\end{abstract}

\section{Introduction}

$\mathrm{CeO}_{2}$ is used in a variety of industrial applications, such as in oxidation catalysis, solid oxide fuel cells, and glass polishing. In many applications (including those mentioned), processes occurring at the surfaces govern $\mathrm{CeO}_{2}$ functionality. For this reason, it would be beneficial to better understand the surface properties of $\mathrm{CeO}_{2}$. Although

\section{R. Stanek}

Material Science and Technology Division, Los Alamos

National Laboratory, Los Alamos, NM 87545, USA

\section{A. H. H. Tan · R. W. Grimes $(\bowtie)$}

Department of Materials, Imperial College, London SW7 2BP,

UK

e-mail: r.grimes@imperial.ac.uk

\section{S. L. Owens}

Nexia Solutions Ltd., Warrington, Cheshire WA3 6AS, UK many studies have addressed $\mathrm{CeO}_{2}$ surface structure and related properties (e.g., [1-4]), relatively few have been concerned with $\mathrm{CeO}_{2}$ surfaces in the presence of water. This is particularly significant to the application of glass polishing because the efficacy of $\mathrm{CeO}_{2}$ abrasive particles depends upon the morphology (or "shape"), which is governed by the relative surface energies of those particles while in the presence of water $[5,6]$.

The mechanism by which glass is polished has been debated for at least 350 years (see [7] and [8] for a more complete description). Despite a long history, it is still the case that no one theory for the mechanism of glass polishing is unequivocally accepted. Rather, three discrete classifications of theory have emerged. Hooke [9], Newton [10], and Rayleigh [11] were all proponents of the so-called "wear theory," which explains glass polishing through the mechanical removal of material by an abrasive polishing agent, and in its simplest terms, is an extension of grinding. Beilby proposed an alternative mechanism [12, 13], known as the "flow theory," where the polishing agent forms an amorphous glass layer that can be smeared to fill voids in the glass surface. Finally, Preston [14] and Grebenshchikov [15] advanced the "chemical theory," which points to the chemical reactions between the polishing agent and the glass surface as the critical component of the polishing process. It is likely that each of these theories play a role in the polishing process, and a generalized "mechano-chemical" mechanism is now nominally accepted $[16,17]$. What remains to be established, however, is whether chemical or mechanical effects dominate.

Only few compounds effectively polish glass, and $\mathrm{CeO}_{2}$ is one of the best $[18,19]$. Interestingly, isostructual $\mathrm{ZrO}_{2}$ has a polishing rate less than half of $\mathrm{CeO}_{2}$ [19]. The maximum polishing activity of $\mathrm{CeO}_{2}$ is achieved by crushing particles sintered at approximately $900{ }^{\circ} \mathrm{C}$ [7]. 
The given reason for this observation is that this sintering temperature yields finer particles which are able to more frequently interact with the glass surface, but it is not so high that the particles sinter together. (Note: Newton also proposed that small particles were more effective at polishing glass, but for the reason that they make smaller scratches on the surface [10]). The optimum preparation route for $\mathrm{CeO}_{2}$ (according to Izumitani [7]) is one that results in a material that is harder than the glass to be polished, but soft enough to be crushed during polishing.

The hardness of the polishing agent is certainly important, but it alone does not completely explain the pronounced efficacy of $\mathrm{CeO}_{2}$. Thus, it has been suggested that chemical reactions between $\mathrm{CeO}_{2}$ and the glass surface also play a role $[14,15]$. Recently, it has been established that the not only the size, but also the shape of the $\mathrm{CeO}_{2}$ crystallites may control the polishing rate [5]. This observation led to subsequent investigations on the effect of synthesis processing on the morphology of $\mathrm{CeO}_{2}$ crystallites [6]. These studies revealed that a more regular distribution of polishing agent particle sizes and morphologies led to more efficient polishing. However, the starting morphology of a $\mathrm{CeO}_{2}$ crystallite should not be expected to remain constant during the polishing process, especially in the presence of water. Furthermore, a morphology of $\mathrm{CeO}_{2}$ crystallites dominated by (111) facets has been proposed as the optimum shape $[5,6]$, but it is not entirely clear why this is so.

In this study, we use atomistic simulation to predict the energy associated with the dissociation of water on the (100), (110), and (111) low index surfaces of $\mathrm{CeO}_{2}$. Consequently, those surfaces which are more strongly stabilized by the presence of hydroxide will be identified. This provides a connection between dissociation of water and particle morphology. Furthermore, based on these results we are able to provide some comments that relate the role of particle morphology to the efficacy of glass polishing.

\section{Previous $\mathrm{CeO}_{2}$ surface simulation studies}

Sayle et al. [20] used similar interatomic potential simulation techniques to those employed in this article to model the surface energies of dry $\mathrm{CeO}_{2}$. In that study, the Type 1 (110) and (310) surfaces (definition of ionic surface type according to Tasker [21]) as well as the Type 2 (111) surface were considered, while the Type 3 (100) surface was not originally considered [20]. Of these, they found the (111) surface to exhibit the lowest surface energy, followed by the (110) and the (310). Conesa [1] expanded on the work of Sayle et al. [20] and examined the stability of the Type 3 (100) surface. To neutralize this dipolar surface, half of the surface oxygen ions were removed (in a checkerboard type configuration).
Using the same potentials as Sayle et al. [20], Conesa found that based upon energetic considerations, the (100) should not be disregarded. Vyas et al. [3] derived a new set of robust potentials, which were able to reproduce the structures of higher index surfaces (e.g., (331), (411), etc.). This work also began to show how $\mathrm{CeO}_{2}$ surface energetics translate to a crystallite morphology. Baudin et al. [22] and Gotte et al. [23] subsequently built upon these static simulations to consider the dynamics of $\mathrm{CeO}_{2}$ surfaces.

Gennard et al. [24] compared results from Hartree-Fock quantum mechanical simulations of bulk and surface properties of $\mathrm{CeO}_{2}$ (and $\mathrm{ZrO}_{2}$ ) to those calculated using interatomic potentials. They found that the energy difference between the (110) and (111) surfaces agreed well with the previous interatomic potential results, though their absolute energies were consistently lower. Skorodumova et al. [4] used density functional theory (both the local density approximation (LDA) and generalized gradient approximation (GGA)) to determine the (111), (110), and (100) surface properties of $\mathrm{CeO}_{2}$. Although the absolute energies were not consistent (even between LDA and GGA), the relative surface energy stability trends remain the same as calculated by the afore-mentioned methods.

In addition to surface stability, the hydroxylation of surfaces has also been simulated. Redfern et al. [25] used interatomic potentials to simulate the hydroxylation of tetragonal- $\mathrm{ZrO}_{2}$ surfaces. The primary conclusion of Redfern et al. [25] was that the equilibrium morphology of tetragonal $\mathrm{ZrO}_{2}$ crystallites is expected to be different in aqueous conditions than in dry conditions. This is because the low-energy surface that dominates this tetragonal crystallite morphology in dry conditions (the (101)) exhibits no tendency to hydroxylate. However, higher energy dry surfaces (e.g., (110)B) exhibit a pronounced tendency to hydroxylate, which then lowers the overall surface energy. The reduction of the (110)B surface energy via hydroxylation increases the stability of this surface so that it is energetically similar to the (101). Similarly, Abramowski et al. [26] and Tan et al. [27] simulated the hydroxylation behavior of $\mathrm{UO}_{2}$ and $\mathrm{PuO}_{2}$, respectively, and again predicted that the crystallite morphology of these compounds is different in the presence of water. Finally, Kumar and Schelling recently considered the adsorption of water on the $\mathrm{CeO}_{2}$ (111) surface via density functional theory [28]. We will compare our results to those of Kumar and Schelling in the "Results" section.

\section{Methodology}

To calculate surface energies, the code MARVIN [29] was employed. As outlined in previous studies [26, 29, 30] MARVIN is a 2D periodic boundary code, where the repeat 
unit is divided into an upper region I and lower region II. All ions in region I are relaxed using energy minimization while those in region II are held fixed at their perfect lattice positions [29]. As is the case with the total depth of the simulation block, regions I and II are chosen so that further enlargement has a negligible effect on total energies (in this study region $\mathrm{I}$ is $3 \times 3 \times 6$ unit cells). MARVIN employs Parry's variant of the Ewald technique $[31,32]$ to sum longrange electrostatic forces. Short-range interactions are described by the Buckingham potential [33]. The potential parameters for $\mathrm{Ce}^{4+}-\mathrm{O}^{2-}$ and $\mathrm{O}^{2-}-\mathrm{O}^{2-}$ can be found in the papers where the original derivation is described ([3] and [34], respectively). Dipoles can be of great significance at surfaces. In addition to the movement of ions, dipoles will also be formed through the displacement of charge around a single ion (i.e., polarization). Here such effects are included by using a core/shell model [35] and the shell parameters are available in the original papers [3, 34].

The methodology described above can be used to calculate two important energies that will characterize the hydroxylation phenomena, namely: surface energy and hydroxylation energy. The surface energy can be defined as

$\gamma=\frac{E_{\text {surf }}-E_{\text {bulk }}}{A}$

where $A$ is the area of the new surface formed, $E_{\text {surf }}$ is the total energy associated with the ions in region I of the $2 \mathrm{D}$ repeat unit and $E_{\text {bulk }}$ is the total energy associated with the same number of ions in a 3D perfect lattice. This is, therefore, the internal energy to cleave a perfect surface and create a new surface. We define hydroxylation energy as the energy gained per water molecule at a specific coverage of water. The method to calculate hydroxylation energies is described elsewhere [25, 26].

The use of fixed interatomic potentials to study surface hydroxylation is a necessary approximation given the large number of possible configurations and large repeat unit cell sizes that must be used to accurately model this problem. Support for the reliability of the interatomic potentials used in this work comes from the similarity of surface-related properties calculated using potentials of this type and

Fig. 1 Three $3 \times 3$ unit cell anion configurations of the $\mathrm{CeO}_{2}$ (100) surface, where the spheres refer to surface oxygen atoms and the unoccupied squares refer to surface vacancies. The nomenclature for these configurations (i.e., A, $\mathrm{B}$, and $\mathrm{AB}$ ) is used throughout the text equivalent surface studies that employ quantum mechanical-based methods as well as experimental observation of crystallite morphology. Several modeling studies for $\mathrm{CeO}_{2}$ were described in the previous section. Another example is the study by Skomurski et al. [36], where the plane wave code CASTEP was used to examine the relative stability of the (111), (110), and (100) surfaces of $\mathrm{UO}_{2}$ (which is isostructural to $\mathrm{CeO}_{2}$ ). Their results agreed favorably with previous results generated with interatomic potentials [30].

\section{Results}

The (111), (110), and (100) surface energies of $\mathrm{CeO}_{2}$ were calculated at $0,12.5,25,50,75$, and $100 \%$ hydroxylation. "Hydroxylation" was simulated by positioning an $\mathrm{OH}^{-}$ group directly above a surface cation and associating the remaining $\mathrm{H}^{+}$of one water molecule with a surface oxygen. All atom positions are then allowed to relax to their equilibrium positions. Consequently, percent hydroxylation is defined as the number of surface cations (or oxygen atoms) covered by water molecules (see [25] for a thorough discussion).

Three configurations of the anion terminated (100) surface (so-called A, B, and $\mathrm{AB}$ ) were considered based upon results of previous studies (see [37] for further details). These three configurations, depicted in Fig. 1, are considered since they are of low energy when compared to other unique configurations of the (100) surface [37]). A trench configuration where (111) facets form grooves on the (100) surface in [110] directions has also been considered (see [27, 37] for additional details of the trench structure). The calculated surface energies of each of these surfaces are described in Table 1. As found in previous theoretical studies [1, 4, 20, 24], the lowest energy dry surface was found to be the (111), followed by the (110) and (100) surfaces. However, as these surfaces become hydroxylated, the energy of the (111) surface increases (becomes less favorable) and at approximately $75 \%$ water coverage actually becomes greater than the surface energies of the (110) and (100) surfaces.

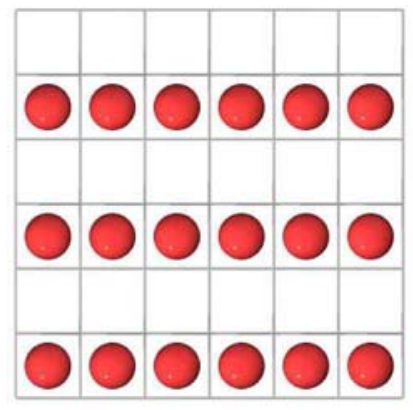

(b) B config.

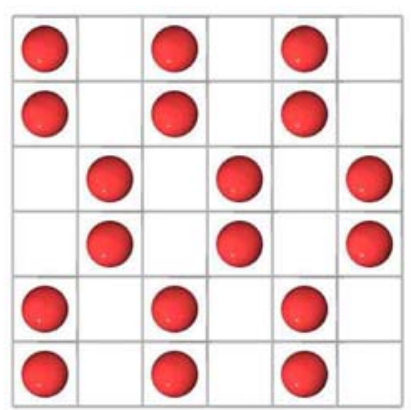

(c) $\mathrm{AB}$ config.

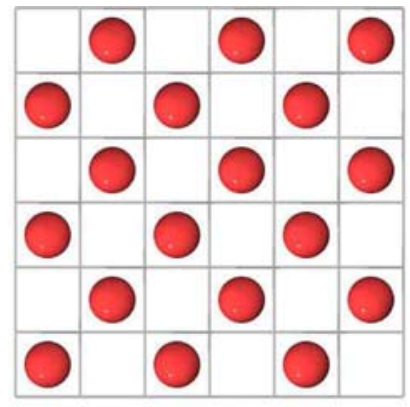

(a) A config. 
Table 1 Surface energies $\left(\mathrm{J} / \mathrm{m}^{2}\right)$ of dry and hydroxylated surfaces of $\mathrm{CeO}_{2}$

\begin{tabular}{lllllll}
\hline$\% \mathrm{H}_{2} \mathrm{O}$ & $(111)$ & $(110)$ & $(100) \mathrm{A}$ & $(100) \mathrm{B}$ & $(100) \mathrm{AB}$ & Trench \\
\hline 0 & 1.39 & 2.17 & 2.94 & 3.30 & 2.88 & 2.56 \\
5.6 & - & - & - & - & - & 2.44 \\
11.1 & - & - & - & - & - & 2.33 \\
12.5 & 1.41 & 2.05 & 2.62 & 2.54 & 2.60 & - \\
16.7 & - & - & - & - & - & 2.25 \\
22.2 & - & - & - & - & - & 2.17 \\
25 & 1.47 & 1.94 & 2.49 & 2.27 & 2.42 & - \\
27.8 & - & - & - & - & - & 2.06 \\
33.3 & - & - & - & - & - & 2.03 \\
38.9 & - & - & - & - & - & 2.08 \\
44.4 & - & - & - & - & - & 2.00 \\
50 & 1.74 & 1.80 & 2.32 & 2.32 & 1.88 & 2.02 \\
55.6 & - & - & - & - & - & 2.04 \\
61.1 & - & - & - & - & - & 2.08 \\
66.7 & - & - & - & - & - & 2.06 \\
72.2 & - & - & - & - & - & 2.05 \\
75 & 1.95 & 1.82 & 1.86 & 1.98 & 1.80 & - \\
77.8 & - & - & - & - & - & 1.89 \\
83.3 & - & - & - & - & - & 2.03 \\
88.9 & - & - & - & - & - & 2.20 \\
94.4 & - & - & - & - & - & 2.37 \\
100 & 3.16 & 1.89 & 1.93 & 1.93 & 1.82 & 1.91 \\
\hline & & & & & &
\end{tabular}

The implication of the (111) surface energy being greater than the hydroxylated (100) surface energy is that the equilibrium $\mathrm{CeO}_{2}$ crystallite morphology will vary depending on the degree of hydroxylation. Figure 2 shows the predicted morphology of $\mathrm{CeO}_{2}$ under dry and low hydroxylation conditions. This morphology is clearly dominated by (111) faces. However, when increasing hydroxylation sufficiently reduces the (100) surface energy (relative to the (111) surface), (100) facets will appear, as shown in Fig. 3. This is consistent with the observation of

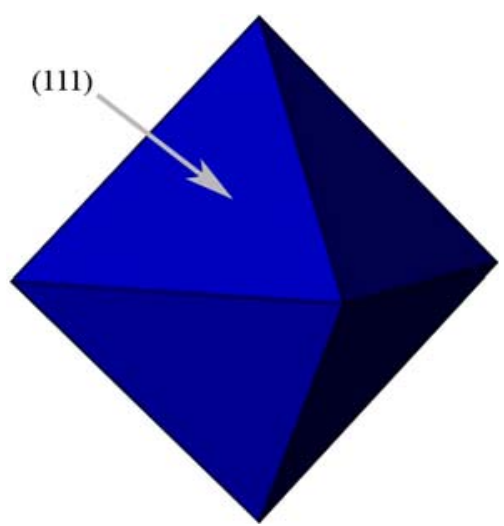

Fig. 2 The predicted morphology of $\mathrm{CeO}_{2}$ crystallites under dry or low hydroxylation conditions
Kirk and Wood [6] who found that the surface area of (100) facets on (111) planes decreased with increasing calcination temperature. Since the $\mathrm{CeO}_{2}$ samples that Kirk and Wood examined were synthesized through a sol-gel process, it is reasonable to expect that water molecules were available for the hydroxylation of surfaces. The reduction in (100) surface area with calcination temperature may suggest that the water molecules stabilizing the (100) surface were removed at the higher temperature. Encouragingly, our prediction of $\mathrm{CeO}_{2}$ morphology in the presence of water (Fig. 3) is nearly identical to the morphology of an approximately $80 \mathrm{~nm} \mathrm{CeO}$ particle observed by Kirk and Wood, after sintering at $850{ }^{\circ} \mathrm{C}[6]$.

Calculated hydroxylation energies for the $\mathrm{CeO}_{2}$ surfaces are plotted as a function of water coverage in Fig. 4, where it is assumed that the hydroxylation energy changes approximately linearly between calculated points. It is clear from Fig. 4 that the hydroxylation energy for the (111) surface is initially close to zero, but becomes more positive (i.e., even less favorable) at greater coverages of water. This suggests that the (111) surface does not exhibit a strong driving force to bind hydroxide species to surface sites. These results compared favourably to the results of Kumar and Schelling [28]. In particular, we calculated a hydroxylation energy of $0.55 \mathrm{eV}$ for the (111) surface at $50 \%$ hydroxylation, while Kumar and Schelling calculated adsorption energies of 0.56 and $0.58 \mathrm{eV}$ for two different configurations of a 0.5 monolayer of water on the (111) surface. However, it should be noted that this comparison is only favorable if our hydroxylation energy is similar to the adsorption energy of Kumar and Schelling and if 50\% hydroxylation corresponds to a 0.5 monolayer.

In contrast to the (111) surface, the (110), (100), and trench surfaces all have negative hydroxylation energies, suggesting that these surfaces will react with available

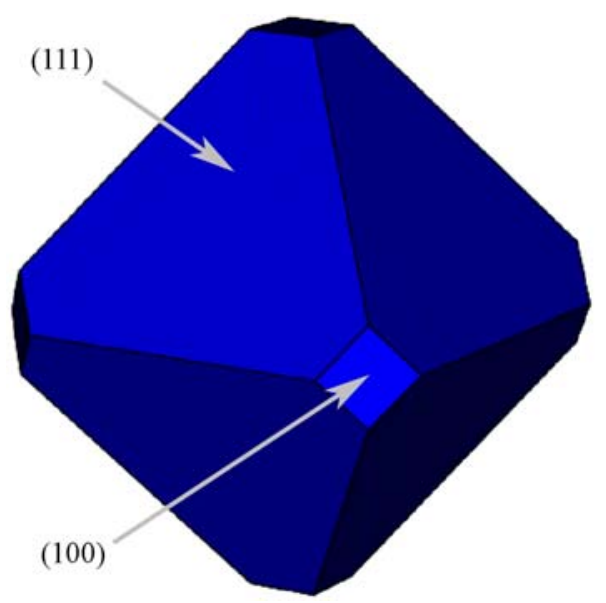

Fig. 3 The predicted morphology of $\mathrm{CeO}_{2}$ crystallites under moderate to high hydroxylation conditions, where the (100) facet surface area is expected to increase as a function of extent of hydoxylation 


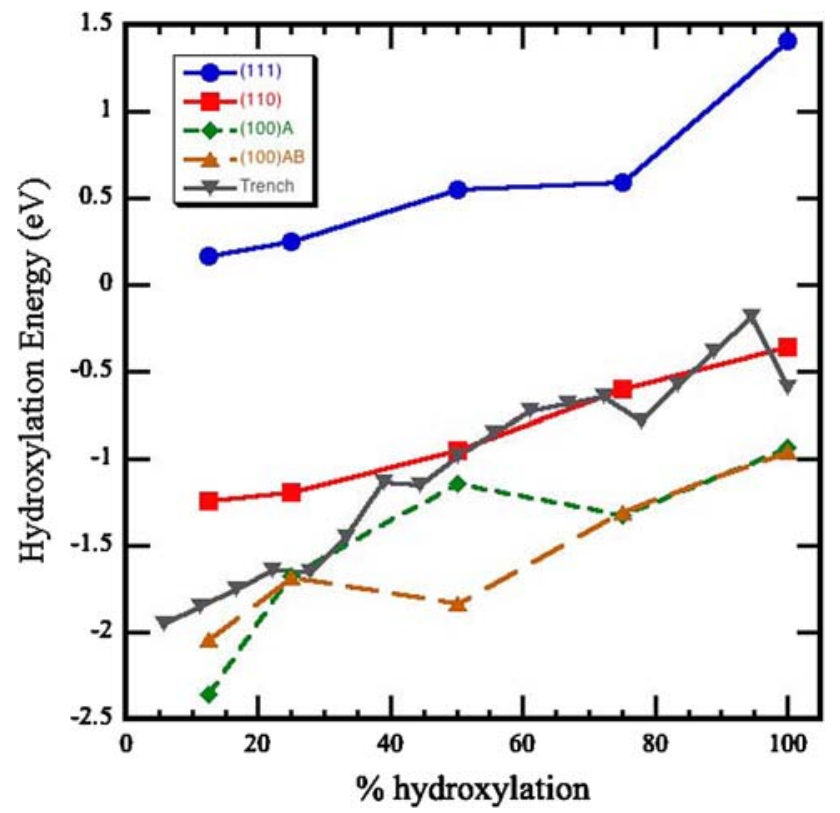

Fig. 4 Comparison of the hydroxylation energies of all $\mathrm{CeO}_{2}$ surfaces considered in this study. Of particular note, the (111) hydroxylation energy is near to but greater than $0 \mathrm{eV}$ for the entire hydroxylation range

water to form stable hydroxylated structures and that hydroxide species are strongly bound to these surfaces. Qualitatively, these predictions can be understood in terms of surface stability and structure of the dry surfaces. The (111) surface is relatively stable when dry, and it is therefore, difficult for hydroxylation to contribute to further stability of the surface. On the other hand, in order for the anion terminated (100) surface to be stable under dry conditions, half of the surface oxygen ions must be removed to neutralize the dipole normal to the surface $[1$, 37]. Since the (100) surface has surface sites that can accommodate the oxygen ions of hydroxide species with a high coordination to adjacent cations, it is not unreasonable to expect that the addition of hydroxide (hydroxylation) will lower the surface energy.

\section{Summary}

We have shown that the equilibrium morphology of $\mathrm{CeO}_{2}$ crystallites will be modified in the presence of water. For glass polishing, it has been recently proposed that in addition to the crystallite size of the glass polishing agent, the shape of the crystallite also plays a role in the efficacy of the glass polishing process [6]. To show this, Kirk and Wood evaluated the polishing efficiency of $\mathrm{CeO}_{2}$ as a function of calcination time and temperature [6]. Izumitani had already shown that the polishing rate of $\mathrm{CeO}_{2}$ starts to decrease when the material is sintered above $900{ }^{\circ} \mathrm{C}$. It is possible that a contributing factor as to why there is a decrease in polishing efficiency for material sintered at higher temperatures is simply that individual $\mathrm{CeO}_{2}$ particles begin to sinter together. Recall, however, that Kirk and Wood found the morphology of $\mathrm{CeO}_{2}$ crystallites calcined at $325^{\circ} \mathrm{C}$ had large (100) facets on (111) planes. When calcined at $850{ }^{\circ} \mathrm{C}$, the surface area of the (100) facets decreased, and the morphology was dominated by (111) faces. Furthermore, this material was found to polish glass rather better, leading Kirk and Wood to conclude that the (111) surface of $\mathrm{CeO}_{2}$ is more effective at polishing glass than the (100) surface [6]. Kirk and Wood suggested the reason for this was that the (111) surface has more $\mathrm{Ce}$ atoms per unit surface (there are $2.31 \mathrm{Ce} / a_{\mathrm{o}}^{2}$ for the (100) surface and $2.00 \mathrm{Ce} / a_{\mathrm{o}}^{2}$ and $1.41 \mathrm{Ce} / a_{\mathrm{o}}^{2}$, respectively, for the (100) and (110) surfaces). This is a plausible explanation if it is the $\mathrm{Ce}^{4+}$ ion that is critical to the mechanochemical process. However, we wish to suggest an alternative possibility. If the chemical process that takes place between $\mathrm{CeO}_{2}$ and the glass surfaces involves transfer of protons or hydroxide species from the $\mathrm{CeO}_{2}$ surface to the glass, then the (111) surface would be more effective than either the (100) or (110) surfaces, simply because the (111) surface does not exhibit a strong affinity for hydroxide species. Indeed, it may even be that the (111) surface provides a source of hydroxide or protons by promoting the dissociation of $\mathrm{H}_{2} \mathrm{O}$ (i.e., catalytic). Certainly, the transfer of hydroxide and protons to the silica, resulting in mulitple $\mathrm{Si}-\mathrm{OH}$ bonds will lead to removal of silicate species material from the glass surface because $\mathrm{Si}(\mathrm{OH})_{4}$ is soluble in water. Moreover, this may explain why $\mathrm{ZrO}_{2}$ is less effective than $\mathrm{CeO}_{2}$, as the (111) surface of $\mathrm{ZrO}_{2}$ has a strongly negative hydroxylation energy [38] and as such will not promote the formation of hydroxide species. Finally, the (111) surface of $\mathrm{UO}_{2}$ also exhibits a clearly negative hydroxylation energy [30], which would be consistent with this material being a less effective polishing agent since the surface will exhibit an energy barrier to the release of a hydroxide or proton. In conclusion, based on the idea that (111) surfaces most readily yield hydroxide or protons that may interact with the glass surface, to maximize the glass polishing efficacy of $\mathrm{CeO}_{2}$, this material should be synthesized such that the morphology of crystallites is dominated by (111) facets, while maintaining small particle size for large overall surface area.

Acknowledgements Los Alamos National Laboratory, an affirmative action/equal opportunity employer, is operated by Los Alamos National Security, LLC, for the National Nuclear Security Administration of the U.S. Department of Energy under contract DE-AC52-06NA25396. Some calculations were performed on the Imperial College High Performance Computing Service, URL: http://www3.imperial.ac.uk/ict/services/ teachingandresearchservices/highperformancecomputing. 
Open Access This article is distributed under the terms of the Creative Commons Attribution Noncommercial License which permits any noncommercial use, distribution, and reproduction in any medium, provided the original author(s) and source are credited.

\section{References}

1. Conesa J (1995) Surf Sci 339:337

2. Sayle T, Parker S, Catlow C (1994) Surf Sci 316:329

3. Vyas S, Grimes R, Gay D, Rohl A (1998) J Chem Soc Faraday Trans $94: 427$

4. Skorodumova N, Baudin M, Hermansson K (2004) Phys Rev B 69:075401

5. Kirk N (1993) Ph.D. thesis, University of Nottingham

6. Kirk N, Wood J (1995) J Mater Sci 30:2171

7. Izumitani T (1988) Optical glass. American Institute of Physics, New York

8. Holland L (1964) The properties of glass surfaces. Chapman and Hall, London

9. Hooke R (1665) Micrographia: or some physiological descriptions of minute bodies made by magnifying glasses with observations and inquiries thereupon. Martyn and Allestry, London

10. Newton I (1704) Opticks: or a treatise of the reflexions, refractions, inflexions and colours of light. Smith and Walford, London

11. Rayleigh J (1901) Nature 64:385

12. Beilby G (1903) Proc Roy Soc A 72:218

13. Beilby G (1921) Aggregation and flow of solids. Macmillan and Co, London
14. Preston F (1930) J Soc Glass Tech 14:127

15. Grebenshchikov I (1931) Keram i Steklo 7:36

16. Cook L (1990) J Non-Cryst Solids 120:152

17. Kirk N, Wood J (1994) Br Ceram Trans 93:25

18. Kaller A (1962) Mschr Feinmech Opt 79

19. Silvernail W, Goetzinger N (1971) Glass Ind 52:130

20. Sayle D, Parker S, Harding J (1994) Philos Mag A 69:787

21. Tasker P (1979) J Phys C 12:4977

22. Baudin M, Wójcik M, Hermansson K (2000) Surf Sci 468:51

23. Gotte A, Hermansson K, Baudin M (2004) Surf Sci 552:273

24. Gennard S, Corà F (1999) J Phys Chem B 103:10158

25. Redfern S, Grimes R, Rawlings R (2001) J Mater Chem 11:449

26. Abramowski M, Redfern S, Grimes R, Owens S (2001) Surf Sci 490:415

27. Tan A, Grimes R, Owens S (2005) J Nucl Mater 344:12

28. Kumar S, Schelling P (2006) J Chem Phys 125:204704

29. Gay D, Rohl A (1995) J Chem Faraday Trans 91:925

30. Abramowski M, Grimes R, Owens S (1999) J Nucl Mater 275:12

31. Parry D (1975) Surf Sci 49:433

32. Parry D (1976) Surf Sci 54:195

33. Buckingham R (1938) Proc Roy Soc A 168:264

34. Binks D, Grimes R (1993) J Am Ceram Soc 76:2370

35. Dick B, Overhauser A (1958) Phys Rev 112:90

36. Skomurski F, Becker U, Ewing R (2004) Geochim Cosmochim Acta 68:A119

37. Tan A, Abramowski M, Grimes R, Owens S (2005) Phys Rev B 72:035457

38. Tan A (2006) Ph.D. thesis Imperial College, London 